\title{
Different Clinical Manifestations of Human Brucellosis in Pregnant Women: A Systematic Scoping Review of 521 Cases from 10 Countries
}

This article was published in the following Dove Press journal: Infection and Drug Resistance

\author{
Zhe Liu' \\ Dawei Wei ${ }^{\text {I }}$ \\ Yanjun Li id \\ Hao Zhou ${ }^{1,2}$ \\ Desheng Huang (D) 1,3 \\ Peng Guan (iD) \\ 'Department of Epidemiology, School of \\ Public Health, China Medical University, \\ Shenyang, Liaoning, People's Republic of \\ China; ${ }^{2}$ Department of Impression \\ Evidence Examination Technology, \\ Criminal Investigation Police University of \\ China, Shenyang, Liaoning, People's \\ Republic of China; ${ }^{3}$ Department of \\ Mathematics, School of Fundamental \\ Sciences, China Medical University, \\ Shenyang, Liaoning, People's Republic of \\ China
}

Introduction: This systematic scoping review aims to assess the frequency and severity of clinical manifestations of pregnant women with brucellosis.

Methods: Three literature databases, PubMed, Web of Science and China National Knowledge Infrastructure (CNKI), and two search engines (Google and Yahoo) were adopted to identify the relevant articles that published until 31 December 2019. Two investigators independently screened the publications and extracted the data; the case reports and case series which described at least two symptoms or clinical manifestations of pregnant women with brucellosis were included.

Results: A total of 27 articles describing the information of 521 pregnant women with brucellosis were included. Serum agglutination test was the most common laboratory test in the diagnosis of brucellosis. A total of 36 clinical manifestations were extracted from the included articles, and the most common clinical manifestations were fever $(400,76.8 \%)$, joint pain/swelling/arthralgia $(389,74.7 \%)$, sweats $(382,73.3 \%)$, fatigue/asthenia/weakness $(262,50.3 \%)$ and back pain $(189,36.3 \%)$. Among the 32 included individual cases that with available obstetric outcome information, 10 (31.3\%) suffered preterm delivery, $12(37.5 \%)$ had an abortion and $3(9.8 \%)$ had intrauterine fetal death.

Conclusion: Brucellosis is popular and threatening for pregnant women. Regarding the localized body system complications, osteoarticular system was mostly involved, the obstetrics outcomes were severe among pregnant women with brucellosis. The detailed clinical and epidemiological characteristics in this scoping review may add a better and more complete understanding of the disease for both physicians and policy-makers, and provide evidence for timely diagnosis, adequate therapy and better prevention.

Keywords: human brucellosis, pregnancy, signs and symptoms, review
Correspondence: Peng Guan

Department of Epidemiology, School of Public Health, China Medical University, Shenyang II0I22, Liaoning, People's Republic of China

Email pguan@cmu.edu.cn

\section{Introduction}

Brucellosis is a worldwide zoonosis that can be encountered during pregnancy, and also classified as one of the "neglected zoonotic diseases" by the World Health Organization and a travel-associated disease, ${ }^{1,2}$ particularly in developing countries. Human brucellosis is mainly caused via three routes, direct contact with secretions and carcasses from Brucella-infected animals, indirect consumption of contaminated products, or inhalation of aerosols. ${ }^{3,4}$ Human-to-human transmission can be via blood transfusion, bone marrow transplantation, and sexual contact. ${ }^{5}$ Neonate can be infected through breast milk and vertical transmission. ${ }^{6}$ There are four species of Brucella that can infect humans, the most pernicious one is Brucella. 
melitensis, which is followed by the Brucella abortus, Brucella suis and Brucella canis. ${ }^{7}$

Brucellosis in pregnancy was first reported in $1908 .{ }^{8}$ The occurrence of brucellosis during pregnancy has been indicated in many countries. In Saudi Arabia, the percentage of brucellosis occurred in pregnant women was reported to be $17 \% .{ }^{9}$ It has been reported that the incidence of brucellosis among 450 pregnant women in Saudi Arabia was $12.2 \%{ }^{10}$ In Rwanda, the brucellosis prevalence among pregnant women was $25.0 \%$, and among the 15 pregnant women diagnosed with brucellosis, $73.3 \%$ had an abortion and 26.7\% presented with stillbirth. ${ }^{11}$ And in Peru, the proportion of fetal death was $8.1 \%$ among 86 women with followup, the percentage of congenital brucellosis and neonatal deaths reached $6.4 \%$ and $11.3 \%$, respectively. ${ }^{12}$

Brucellosis has a wide spectrum of clinical manifestations, once infected with brucellosis, most patients complained about a series of flu-like symptoms such as fever, sweats, fatigue, headache, myalgia and arthralgia. ${ }^{13}$ However, there are still various atypical symptoms that can cause misdiagnosis easily, especially for the pregnant women. Adverse obstetric complications were usually recorded as abortion (spontaneous or therapeutic), premature delivery, intrauterine infection or intrauterine fetal death (IUFD) for pregnant women diagnosed with brucellosis. In some studies, it has been demonstrated that pregnant women with brucellosis had a higher morbidity of spontaneous abortion than the general population of pregnant women. ${ }^{9,10}$ However, it remains disputed about the relationship between brucellosis and the other adverse outcomes of pregnancy. ${ }^{14}$ Also, it is unknown whether brucellosis causes a higher incidence of abortion than the infection of other bacteria. ${ }^{15}$

Considering that there were only a few studies concentrating on the clinical manifestations of pregnant women with brucellosis, we reviewed the relevant studies in order to assess the frequency and severity of the clinical manifestations and investigate the main risk factors for pregnant women with brucellosis. The clinical and epidemiological results in the systemic review would allow people to know more details about human brucellosis during pregnancy.

\section{Materials and Methods}

\section{Literature Search Strategy}

A thorough literature search was conducted through PubMed, Web of Science, and China National Knowledge
Infrastructure (CNKI) to identify the studies published until 31 December 2019 and reporting clinical manifestations of brucellosis in pregnancy, using search terms of (brucellosis OR malta fever OR brucellosis malitensis OR brucellosis abortus) AND pregnancy without language restriction. Reference lists of the included studies were screened manually to trace the relevant articles, the internet search engines of both Google and Yahoo were also adopted to search for the relevant publications. The search results were independently screened and extracted by two investigators (ZL and DW), and all the discrepancies were resolved by the principal investigator (PG). Results were imported into EndNote X7 and duplicates were removed after reading the author, title, abstract, year of publication and source of the article.

\section{Inclusion and Exclusion Criteria}

As long as the publication contained individual patient information that can be extracted and the number of cases was no more than 10 , it was defined as a case report, and if there was only aggregated information that could be obtained in the article or the number of individual cases was more than 10 , it was defined as the case series. Case reports and case series studies were eligible for the inclusion if they met the following criteria, (a) reporting one or more pregnant women diagnosed with brucellosis; (b) reporting at least two symptoms or clinical manifestations; (c) all the included cases were diagnosed as brucellosis by at least one of the following laboratory tests: blood/bone marrow culture, serum agglutination test (SAT), rose bengal plate agglutination test (RBT), enzyme-linked immunosorbent assay (ELISA), 2-mercaptoethanol (2ME), polymerase chain reaction (PCR), complement fixation test (CFT), anti-human immunoglobulin test (Coomb's test), and blocking antibody titer (BAB). Articles were excluded if they met one of the following conditions: (a) articles related to nonhuman brucellosis after reading the title and abstract; (b) articles referred to brucellosis while without relevance to pregnancy; (c) no description of clinical manifestations or symptoms of pregnant women with brucellosis in its abstract and main-text; (d) duplicated data, and the article with smaller sample size was removed.

\section{Data Extraction}

We extracted the information as follows: author, country of origin of patients, published year, age of the patient at the time of presentation, trimester of pregnancy, clinical manifestations, the duration of delayed diagnosis and the 
obstetric outcomes. The first trimester of pregnancy was defined as a gestational period of $\leq 12$ weeks; the second trimester of pregnancy, 12-24 weeks; and the third trimester of pregnancy, $\geq 25$ weeks. Fetal death that occurred at less than 24 weeks of gestation was considered to be spontaneous abortion. Fetal death that occurred at 24 weeks of gestation or more was designated "intrauterine fetal death" (IUFD). Preterm delivery was defined as the birth of a baby before 37 weeks but after 24 weeks of gestation. ${ }^{16}$ The duration of delayed diagnosis referred to the duration from the onset of any symptoms to the final definite diagnosis of the disease, we can speculate it according to the medical history. Clinical manifestation was defined as patient-level finding gathered during medical interview, physical examination, or through diagnostic studies. The data were extracted into a Microsoft Excel sheet and organized into system-wide manifestations of brucellosis in pregnancy. Descriptive statistics were used to calculate the simple frequency, percentage and proportion of the total subjects.

\section{Data Synthesis}

We defined an event rate as the ratio of the number of reported cases with a specific clinical manifestation to the total number of the reported cases in each study. The qualitative data were described as number and proportion (\%). Quantitative variables were described as mean and standard deviation.

\section{Results}

\section{Characteristics and Main Results of the Included Studies}

A total of 1143 publications were found based on the search strategy, and then 873 articles were screened after removing 270 duplicates by reading the titles, authors, published year and sources. After reading the titles and abstracts, we removed 613 articles and sent 260 articles to assess the eligibility, and 233 articles were excluded after reading the paper. Finally, 27 publications met the inclusion criteria; among them, seven publications reported case series with the information of 488 pregnant women with brucellosis and 20 publications were case reports with individual information of 33 pregnant women with brucellosis. More than half of the articles were from Turkey and China, and 17 articles (63.0\%) were published after 2010. Figure 1 illustrates the detailed process of selection of the included articles according to the
Preferred Reporting Items for Systematic Reviews and MetaAnalyses (PRISMA). ${ }^{17}$

Table 1 shows the main results extracted from the case series and case reports. A total of 521 cases of pregnant women with laboratory-confirmed brucellosis were reported in the included 27 publications. The median of sample size of these seven case series studies was 33, ranging from 19 to 242 . The mean/median maternal ages were basically the same (about 28 years old, respectively), ranging from 16 to 50 years. Pregnant women presented symptoms across all the trimesters, the proportion of pregnant women with brucellosis presented symptoms at the first trimester ranged from $12.9 \%$ to $57.9 \%$, while it ranged from $22.2 \%$ to $51.6 \%$ at the second trimester. The most commonly used laboratory tests for brucellosis were serum agglutination test (SAT) and blood/bone marrow culture, which were used in $85.7 \%$ and $71.4 \%$ studies among the case series, respectively, and $66.7 \%$ and $45.5 \%$ cases were diagnosed with brucellosis using SAT and blood/bone marrow culture respectively among the case reports. The incidence of brucellosis occurred in pregnant women ranged from $1.5 \%$ to $5.8 \%$. As for the adverse obstetric outcomes, the rate of abortion ranged from $2.5 \%$ to $53.0 \%$, the rate of preterm delivery ranged from $3.4 \%$ to $31.3 \%$ and the IUFD rate ranged from $2.1 \%$ to $9.8 \%$.

Most pregnant women with brucellosis had a history of consumption of cheese or non-pasteurized dairy products (92.3\% in the study by Kurdoglu et al and $90.8 \%$ in the study by Vilchez et al) or living in a low socioeconomic class or rural areas. There were no neonates infected with brucellosis in the included case series study in Turkey, while the rate of congenital brucellosis was $6.4 \%$ in the included case series study in Peru. Regarding the severity of brucellosis, a 31-week pregnant woman who presented with a 3-week history of the disease died on the disseminated intravascular coagulation (DIC) in the study by Vilchez et al. $^{12}$

\section{Clinical Manifestations and Obstetric Outcomes}

Table 2 shows the detailed clinical manifestations, diagnostic methods and obstetric outcomes of pregnant women with brucellosis, which were extracted from the included case reports. The duration of delayed diagnosis could only be speculated for eight cases, ranging from 7 days to 5 months. 


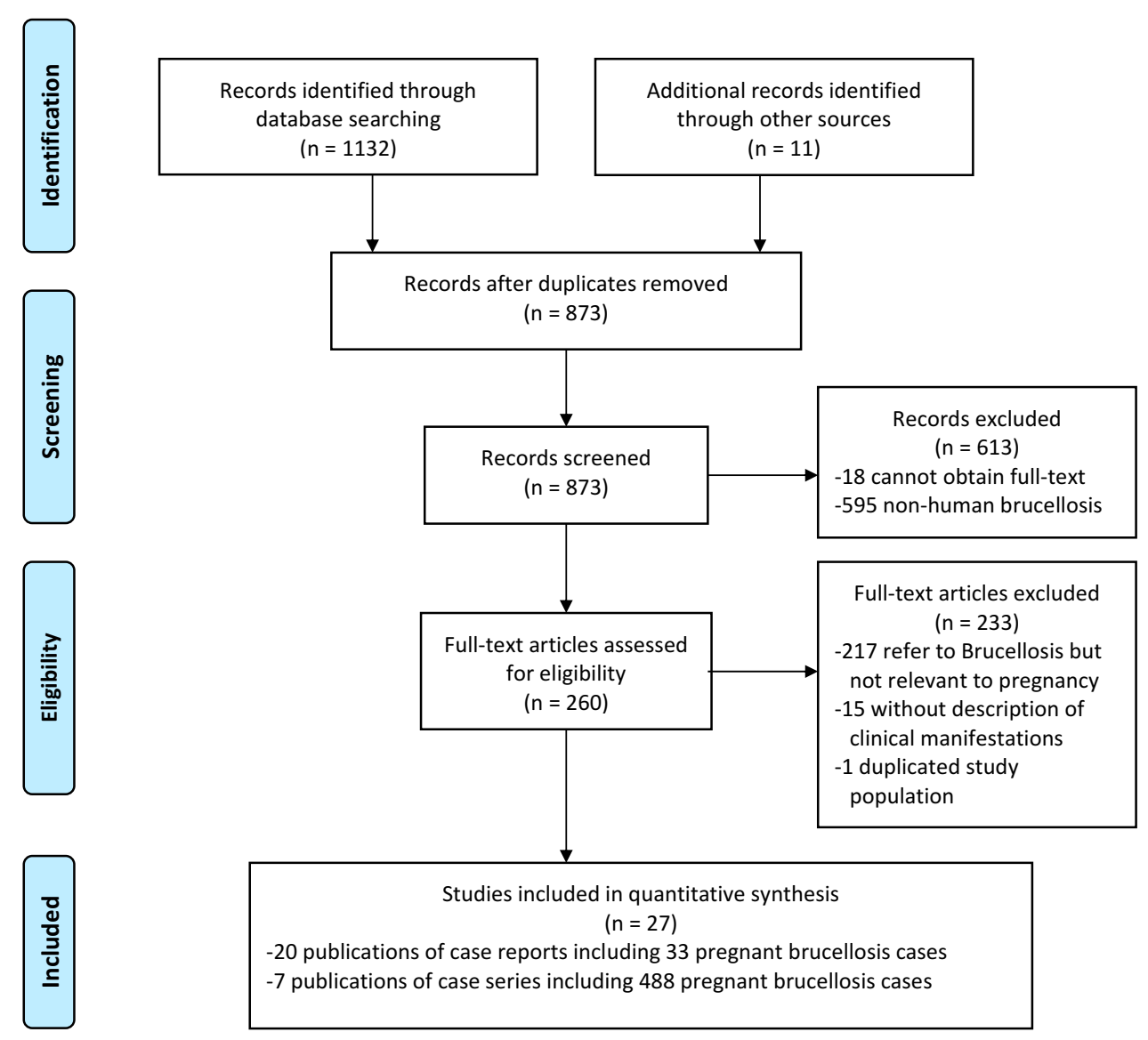

Figure I Flow diagram of selection of studies included in the review.

And we recorded the clinical manifestations as the frequency of occurrence and percentage (\%) in the descending order in Table 3, the pooled proportions were calculated. A total of 36 clinical manifestations were extracted from the included articles, and the most common clinical manifestations were fever (400, 76.8\%), joint pain/swelling/arthralgia (389, 74.7\%), sweats $(382,73.3 \%)$, fatigue/asthenia/weakness $(262,50.3 \%)$ and back pain $(189,36.3 \%)$. Among the seven case series, the study by Vilchez et al reported the largest varieties of clinical manifestations (20/36, 55.6\%), and the top three were fever $(97.0 \%)$, malaise $(85.1 \%)$ and sweats (77.2\%). Among the 33 individual cases, a total of 29 (85.3\% of 36) kinds of clinical manifestations were reported, the most common clinical manifestations were fever (66.6\%), sweats (51.5\%), joint pain/swelling/arthralgia (39.4\%) and vaginal bleeding (24.2\%). There were five (13.7\% of 36$)$ kinds of clinical manifestations that were only reported in the case reports, such as membrane rupture, oligohydramnios and breast pain, while there were no records about such as constipation, rash and depression in the included case reports. It is worth mentioning that one patient from the included case reports complained of both auditory hallucinations, dull/delirious, hand tremors and insomnia.

As for the clinical manifestations of the involved body systems among pregnant women with brucellosis, osteoarticular system was mostly involved, $74.7 \%$ of the pregnant women with brucellosis presented joint pain/swelling/arthralgia. A proportion of $12.3 \%$ of the patients presented the symptoms of cardiovascular system as hypoxia. For the clinical manifestations of reproductive system, vaginal bleeding $(9.0 \%)$ was the most common clinical manifestation, followed by abdominal pain/distension/pelvic pain (7.5\%).

Among the 32 included individual cases that with available obstetric outcome information, 10 (31.3\%) women suffered preterm delivery, 12 (37.5\%) had an abortion and $3(9.8 \%)$ had intrauterine fetal death. And among the 12 patients who had an abortion, $5(41.7 \%)$ of them experienced vaginal bleeding.

\section{Discussion}

Brucellosis is an epidemic zoonosis in developing countries that can affect any organs and body systems and 
Table I Overview of the Included Seven Case Series Studies and 20 Case Reports of Brucellosis in Pregnant Women

\begin{tabular}{|c|c|c|c|c|c|c|c|c|}
\hline \multirow{2}{*}{$\begin{array}{l}\text { First Author, } \\
\text { Publication } \\
\text { Year }\end{array}$} & \multicolumn{7}{|c|}{ From Seven Case Series Studies (\%) } & \multirow{2}{*}{$\begin{array}{l}\text { Case } \\
\text { Reports } \\
48\end{array}$} \\
\hline & $\begin{array}{l}\text { Madkour } \\
\text { MM }(2001)^{49}\end{array}$ & $\begin{array}{l}\text { Kurdoglu } \\
M(2010)^{23}\end{array}$ & $\begin{array}{l}\text { Gulsun } \\
\text { S }(2011)^{16}\end{array}$ & $\begin{array}{l}\text { Roushan } \\
\text { MR } \\
(20 I I)^{20}\end{array}$ & $\begin{array}{l}\text { Vilchez } \\
\text { G }(2015)^{12}\end{array}$ & $\begin{array}{l}\text { Ali } \\
\text { S }(20 \mid 6)^{50}\end{array}$ & $\begin{array}{l}\text { Inan } \\
\text { A }(2019)^{51}\end{array}$ & \\
\hline $\begin{array}{l}\text { Country, time } \\
\text { of study }\end{array}$ & $\begin{array}{l}\text { Saudi Arabia } \\
\text { (before 200I) }\end{array}$ & $\begin{array}{l}\text { Turkey } \\
2003-2008\end{array}$ & $\begin{array}{l}\text { Turkey } \\
2003-2010\end{array}$ & $\begin{array}{l}\text { Iran } \\
2000-2010\end{array}$ & $\begin{array}{l}\text { Peru } \\
1970-2012\end{array}$ & $\begin{array}{l}\text { Pakistan } \\
2013\end{array}$ & $\begin{array}{l}\text { Turkey } \\
2002-2015\end{array}$ & + \\
\hline $\begin{array}{l}\text { Number of } \\
\text { patients } \\
\text { involved }\end{array}$ & 29 & 33 & 39 & 19 & 101 & 25 & 242 & 33 \\
\hline $\begin{array}{l}\text { Mean/median } \\
\text { age (range of } \\
\text { age, years) }\end{array}$ & $\begin{array}{l}29.2 \pm 6.2 \\
(16-4 I)\end{array}$ & $\begin{array}{l}26.8 \pm 5.1 \\
(16-42)\end{array}$ & $\begin{array}{l}28.07 \\
(20-40)\end{array}$ & $\begin{array}{l}25 \pm 4.6 \\
(18-36)\end{array}$ & $\begin{array}{l}26.1 \\
(15-45)\end{array}$ & $\begin{array}{l}27 \\
(|8-4|+)\end{array}$ & $\begin{array}{l}28.8 \pm 6.3 \\
(17-50)\end{array}$ & $\begin{array}{l}28.2 \pm 5.8 \\
(16-39)\end{array}$ \\
\hline Trimester (\%) & $\begin{array}{l}\text { First (4I.4) } \\
\text { Second (24.I) } \\
\text { Third (34.5) }\end{array}$ & $\begin{array}{l}\text { First (39.4) } \\
\text { Second } \\
(33.3) \\
\text { Third (27.3) }\end{array}$ & $\begin{array}{l}\text { Not } \\
\text { mentioned }\end{array}$ & First (57.9) & $\begin{array}{l}\text { First }(45.5) \\
\text { Second }(22.2) \\
\text { Third }(32.3)\end{array}$ & $\begin{array}{l}\text { Not } \\
\text { mentioned }\end{array}$ & $\begin{array}{l}\text { First (24.8) } \\
\text { Second } \\
(39.3) \\
\text { Third (32.2) }\end{array}$ & $\begin{array}{l}\text { First (12.9) } \\
\text { Second (5I.6) } \\
\text { Third (35.2) }\end{array}$ \\
\hline $\begin{array}{l}\text { Diagnostic } \\
\text { methods }\end{array}$ & $\begin{array}{l}\text { SAT, blood } \\
\text { culture }\end{array}$ & $\begin{array}{l}\text { SAT, } \\
\text { Coomb's } \\
\text { test }\end{array}$ & $\begin{array}{l}\text { SAT, } \\
\text { Coomb's, } \\
\text { blood } \\
\text { culture }\end{array}$ & $\begin{array}{l}\text { SAT, 2ME, } \\
\text { blood } \\
\text { culture }\end{array}$ & $\begin{array}{l}\text { SAT, BAB, } \\
\text { blood/bone } \\
\text { marrow } \\
\text { culture }\end{array}$ & RBPT & $\begin{array}{l}\text { SAT, RBPT, } \\
\text { blood } \\
\text { culture, } \\
\text { Coomb's }\end{array}$ & $\dagger$ \\
\hline $\begin{array}{l}\text { Incidence of } \\
\text { brucellosis in } \\
\text { pregnancy (\%) }\end{array}$ & 5.8 & $\begin{array}{l}\text { Not } \\
\text { mentioned }\end{array}$ & $\begin{array}{l}\text { Not } \\
\text { mentioned }\end{array}$ & 1.5 & $\begin{array}{l}\text { Not } \\
\text { mentioned }\end{array}$ & 5.8 & 2.1 & $\begin{array}{l}\text { Not } \\
\text { applicable }\end{array}$ \\
\hline $\begin{array}{l}\text { Adverse } \\
\text { outcomes (\%) }\end{array}$ & $\begin{array}{l}\text { Abortion } \\
(4 I .0), \\
\text { Preterm } \\
\text { delivery (3.4) } \\
\text { IUFD (3.4) }\end{array}$ & $\begin{array}{l}\text { Abortion } \\
(24.1) \\
\text { Preterm } \\
\text { delivery } \\
(6.9) \\
\text { IUFD (3.5) }\end{array}$ & $\begin{array}{l}\text { Abortion } \\
(2.5 \%) \\
\text { Preterm } \\
\text { deliveries } \\
\text { (17.9\%); }\end{array}$ & $\begin{array}{l}\text { Abortion } \\
(53.0)\end{array}$ & $\begin{array}{l}\text { Preterm } \\
\text { delivery (13.9) } \\
\text { Spontaneous } \\
\text { abortion } \\
(12.8) \\
\text { IUFD (8.I) }\end{array}$ & $\begin{array}{l}\text { Not } \\
\text { mentioned }\end{array}$ & $\begin{array}{l}\text { Preterm } \\
\text { delivery (1.2) } \\
\text { Abortion } \\
(6.2) \\
\text { IUFD (2.I) }\end{array}$ & $\begin{array}{l}\text { Preterm } \\
\text { delivery (3I.3) } \\
\text { Abortion } \\
(37.5) \\
\text { IUFD (9.8) }\end{array}$ \\
\hline $\begin{array}{l}\text { Epidemiological } \\
\text { history (\%) }\end{array}$ & $\begin{array}{l}\text { Not } \\
\text { mentioned }\end{array}$ & $\begin{array}{l}A(92.3) \\
B(58.8) \\
C(63.2) \\
D(50.0) \\
E(80.0) \\
F(60.0)\end{array}$ & $\begin{array}{l}C(76.9) \\
E(46.1)\end{array}$ & $\begin{array}{l}A(63.2) \\
B(I 5.8)\end{array}$ & A (90.8) & $\begin{array}{l}A(52.0) \\
B(36.0) \\
E(68.0)\end{array}$ & & $\begin{array}{l}A(18.5) \\
C / D(22.2) \\
E(44.4)\end{array}$ \\
\hline
\end{tabular}

Notes: A, consumption of unpasteurized or infected dairy products or meat; B, stockbreeding or contact with animals; C, previous diagnosis in family members; D, previous diagnosis in neighbors; $\mathrm{E}$, low socioeconomic class or rural areas; $\mathrm{F}$, not literate. ${ }^{\dagger}$ Detailed in Table 2.

Abbreviations: 2ME, 2-mercaptoethanol; BAB, blocking antibody titer; Coomb's test, anti-human immunoglobulin test; IUFD, intrauterine fetal death; RBPT, rose bengal plate agglutination test; SAT, serum agglutination test.

mimic other diseases. Although several studies are available regarding the clinical features of human brucellosis, ${ }^{18}$ the clinical manifestations of brucellosis among pregnant population and the geographical distribution are limited. This is the first systematic scoping review characterizing the clinical manifestations of pregnant women with human brucellosis.
Of the 27 included studies, all the reported pregnant women with brucellosis yield in this systemic review were originally from Mediterranean countries, Asia, America and the Nordic, this is consistent with another review. ${ }^{19}$ In view of the fact that most of the included publications were published after 2000, it can be speculated that brucellosis remains epidemic and the pregnant women are at 
Table 2 Clinical Manifestations of Individual Case Reports of Brucellosis in Pregnant Women

\begin{tabular}{|c|c|c|c|c|c|c|c|c|}
\hline $\begin{array}{l}\text { Patient } \\
\text { No. }\end{array}$ & Reference & $\begin{array}{l}\text { Age } \\
\text { (Years) }\end{array}$ & Country & Trimester & $\begin{array}{l}\text { Clinical } \\
\text { Manifestations }\end{array}$ & $\begin{array}{l}\text { Diagnostic } \\
\text { Methods }\end{array}$ & $\begin{array}{l}\text { Duration of } \\
\text { Delayed } \\
\text { Diagnosis }\end{array}$ & $\begin{array}{l}\text { Obstetric } \\
\text { Outcomes }\end{array}$ \\
\hline I & Sarram M $(1974)^{30}$ & 35 & Iran & Second & $\begin{array}{l}\text { Fever, headache, } \\
\text { sweating, malaise, }\end{array}$ & $\begin{array}{l}\text { SAT } \\
\text { Blood culture }\end{array}$ & Not mentioned & Abortion \\
\hline 2 & Sarram M $(1974)^{30}$ & 25 & Iran & Second & Joint pains & SAT & Not mentioned & Abortion \\
\hline 3 & Sarram M $(1974)^{30}$ & 17 & Iran & Second & $\begin{array}{l}\text { Muscle and bone } \\
\text { pains }\end{array}$ & $\begin{array}{l}\text { SAT } \\
\text { Blood culture }\end{array}$ & Not mentioned & Abortion \\
\hline 4 & Sarram M (1974) 30 & 25 & Iran & Second & Joint pains & $\begin{array}{l}\text { SAT } \\
\text { Blood culture }\end{array}$ & Not mentioned & Abortion \\
\hline 5 & Sarram M $(1974)^{30}$ & 21 & Iran & Second & $\begin{array}{l}\text { Fever, sweating, joint } \\
\text { pains }\end{array}$ & $\begin{array}{l}\text { SAT } \\
\text { Blood culture }\end{array}$ & Not mentioned & Abortion \\
\hline 6 & Sarram M $(1974)^{30}$ & 25 & Iran & Second & $\begin{array}{l}\text { Sweating, muscle, } \\
\text { joint pains }\end{array}$ & SAT & Not mentioned & Abortion \\
\hline 7 & Schreyer $(1980)^{25}$ & 35 & Israel & Second & $\begin{array}{l}\text { Fever, chills, malaise, } \\
\text { nausea, lower } \\
\text { abdominal pains }\end{array}$ & Blood culture & Not mentioned & IUFD \\
\hline 8 & Naveau $(1983)^{31}$ & 34 & USA & Second & $\begin{array}{l}\text { Anorexia, fatigue, } \\
\text { weight loss }\end{array}$ & CFT & Not mentioned & $\begin{array}{l}\text { Term } \\
\text { delivery }\end{array}$ \\
\hline 9 & Gloeb $(1994)^{32}$ & 24 & USA & Third & $\begin{array}{l}\text { Fever, shaking chills, } \\
\text { night sweating, } \\
\text { weakness, anorexia, } \\
\text { nausea, vomiting, } \\
\text { vague pain, upper } \\
\text { abdominal pain, } \\
\text { a non-productive } \\
\text { cough, hand tremors, } \\
\text { auditory } \\
\text { hallucinations }\end{array}$ & SAT & 8 days & $\begin{array}{l}\text { Preterm } \\
\text { delivery }\end{array}$ \\
\hline 10 & $\operatorname{Dan}(200 \mathrm{I})^{33}$ & 34 & China & Third & $\begin{array}{l}\text { Fever, sweating, } \\
\text { general joint pain to } \\
\text { knee joint clear, } \\
\text { paroxysmal irregular } \\
\text { abdominal pain }\end{array}$ & SAT & 8 days & $\begin{array}{l}\text { Preterm } \\
\text { delivery }\end{array}$ \\
\hline 11 & $\operatorname{Dan}(200 \mathrm{I})^{33}$ & 27 & China & Second & $\begin{array}{l}\text { Fever, sweating, } \\
\text { fatigue, general joints } \\
\text { and muscles pain, } \\
\text { insomnia }\end{array}$ & ELISA & Not mentioned & IUFD \\
\hline 12 & Jensenius $(2008)^{34}$ & 33 & Norway & Third & $\begin{array}{l}\text { Fever, both breasts } \\
\text { pain }\end{array}$ & $\begin{array}{l}\text { Blood culture } \\
\text { ELISA }\end{array}$ & Not mentioned & $\begin{array}{l}\text { Not } \\
\text { mentioned }\end{array}$ \\
\hline 13 & Cebesoy $(2009)^{35}$ & 21 & Turkey & First & Fever, back pain & $\begin{array}{l}\text { SAT } \\
\text { Blood culture }\end{array}$ & Not mentioned & $\begin{array}{l}\text { Term } \\
\text { delivery } \\
\text { (caesarean) }\end{array}$ \\
\hline 14 & $\begin{array}{l}\text { Karcaaltincaba } \\
(2010)^{36}\end{array}$ & 25 & Turkey & Second & $\begin{array}{l}\text { Fever, sweats, } \\
\text { backache, malaise }\end{array}$ & $\begin{array}{l}\text { Bone } \\
\text { marrow } \\
\text { culture }\end{array}$ & Not mentioned & $\begin{array}{l}\text { Term } \\
\text { delivery }\end{array}$ \\
\hline 15 & $\begin{array}{l}\text { Karcaaltincaba } \\
(2010)^{36}\end{array}$ & 33 & Turkey & Second & $\begin{array}{l}\text { Fever, sweating, } \\
\text { vaginal bleeding, } \\
\text { rupture of the } \\
\text { membranes }\end{array}$ & $\begin{array}{l}\text { SAT } \\
\text { Blood culture }\end{array}$ & Not mentioned & Abortion \\
\hline
\end{tabular}

(Continued) 
Table 2 (Continued).

\begin{tabular}{|c|c|c|c|c|c|c|c|c|}
\hline $\begin{array}{l}\text { Patient } \\
\text { No. }\end{array}$ & Reference & $\begin{array}{l}\text { Age } \\
\text { (Years) }\end{array}$ & Country & Trimester & $\begin{array}{l}\text { Clinical } \\
\text { Manifestations }\end{array}$ & $\begin{array}{l}\text { Diagnostic } \\
\text { Methods }\end{array}$ & $\begin{array}{l}\text { Duration of } \\
\text { Delayed } \\
\text { Diagnosis }\end{array}$ & $\begin{array}{l}\text { Obstetric } \\
\text { Outcomes }\end{array}$ \\
\hline 16 & Peker $(20 \mathrm{II})^{37}$ & 19 & Turkey & Second & $\begin{array}{l}\text { Fever, malaise, } \\
\text { hematuria, nausea, } \\
\text { membrane rupture }\end{array}$ & Blood culture & Not mentioned & Abortion \\
\hline 17 & Ceylan $(2012)^{38}$ & 18 & Turkey & Third & $\begin{array}{l}\text { Malaise, anorexia, } \\
\text { intermittent vomiting, } \\
\text { vaginal bleeding }\end{array}$ & SAT & 2 months & $\begin{array}{l}\text { Preterm } \\
\text { delivery }\end{array}$ \\
\hline 18 & $\mathrm{Bi}(2013)^{39}$ & 28 & China & Third & $\begin{array}{l}\text { Left lower limb pain, } \\
\text { fever, sweating, } \\
\text { paroxysmal abdominal } \\
\text { pain }\end{array}$ & RBPT & II days & $\begin{array}{l}\text { Preterm } \\
\text { delivery } \\
\text { (caesarean } \\
\text { section) }\end{array}$ \\
\hline 19 & Aydın $(2013)^{40}$ & 27 & Turkey & Second & $\begin{array}{l}\text { Sweating, arthralgia, } \\
\text { oligohydramnios, } \\
\text { premature rupture of } \\
\text { membranes }\end{array}$ & SAT & Not mentioned & $\begin{array}{l}\text { Preterm } \\
\text { delivery }\end{array}$ \\
\hline 20 & $\mathrm{Li}(2015)^{41}$ & 34 & China & First & $\begin{array}{l}\text { Fever, cough, vaginal } \\
\text { bleeding }\end{array}$ & Blood culture & Not mentioned & Abortion \\
\hline 21 & Fathassi $(2016)^{42}$ & 30 & Tunisia & Second & $\begin{array}{l}\text { Fever, myalgia, } \\
\text { arthralgia, asthenia, } \\
\text { nausea, vomiting }\end{array}$ & SAT & Not mentioned & $\begin{array}{l}\text { Term } \\
\text { delivery }\end{array}$ \\
\hline 22 & Fathassi $(2016)^{42}$ & 39 & Tunisia & Third & $\begin{array}{l}\text { Fever, pelvic pain } \\
\text { (uterine } \\
\text { contractions), } \\
\text { membrane rupture }\end{array}$ & SAT & Not mentioned & $\begin{array}{l}\text { Preterm } \\
\text { delivery }\end{array}$ \\
\hline 23 & Agah $(2016)^{43}$ & 29 & Iran & First & $\begin{array}{l}\text { Fever, chills, painless } \\
\text { vaginal bleeding with } \\
\text { symptoms of partial } \\
\text { abortion }\end{array}$ & $\begin{array}{l}\text { SAT } \\
2 \mathrm{ME}\end{array}$ & 10 days & Abortion \\
\hline 24 & Agah $(2016)^{43}$ & 31 & Iran & First & $\begin{array}{l}\text { Fever, chills, night } \\
\text { sweating, low back } \\
\text { pain, vaginal bleeding }\end{array}$ & $\begin{array}{l}\text { SAT } \\
2 \mathrm{ME}\end{array}$ & 7 days & Abortion \\
\hline 25 & Agah $(2016)^{43}$ & 36 & Iran & Second & $\begin{array}{l}\text { Shaking chills, day } \\
\text { time sweating, vaginal } \\
\text { bleeding }\end{array}$ & $\begin{array}{l}\text { SAT } \\
2 M E\end{array}$ & Not mentioned & IUFD \\
\hline 26 & Agah $(2016)^{43}$ & 36 & Iran & Third & $\begin{array}{l}\text { Long-term backache, } \\
\text { malaise, sweating }\end{array}$ & $\begin{array}{l}\text { SAT } \\
2 M E\end{array}$ & Not mentioned & $\begin{array}{l}\text { Term } \\
\text { delivery }\end{array}$ \\
\hline 27 & Agah $(2016)^{43}$ & 32 & Iran & Third & $\begin{array}{l}\text { Right shoulder pain, } \\
\text { sweating }\end{array}$ & $\begin{array}{l}\text { SAT } \\
2 \mathrm{ME}\end{array}$ & 10 weeks & $\begin{array}{l}\text { Term } \\
\text { delivery }\end{array}$ \\
\hline 28 & Zhou $(2017)^{44}$ & 26 & China & Third & $\begin{array}{l}\text { Fever, chills, sweating, } \\
\text { asthenia }\end{array}$ & $B A B$ & Not mentioned & $\begin{array}{l}\text { Preterm } \\
\text { delivery } \\
\text { (caesarean } \\
\text { section) }\end{array}$ \\
\hline 29 & Zhou $(2017)^{44}$ & 25 & China & Third & $\begin{array}{l}\text { Fever, sweating, } \\
\text { asthenia, vaginal } \\
\text { bleeding with } \\
\text { abdominal pain }\end{array}$ & $\mathrm{BAB}$ & Not mentioned & $\begin{array}{l}\text { Preterm } \\
\text { delivery } \\
\text { (caesarean } \\
\text { section) }\end{array}$ \\
\hline 30 & Yang $(2018)^{45}$ & 22 & China & $\begin{array}{l}\text { Not } \\
\text { mentioned }\end{array}$ & $\begin{array}{l}\text { Fever, vaginal } \\
\text { bleeding, abdominal } \\
\text { distension }\end{array}$ & $\begin{array}{l}\text { SAT } \\
\text { Blood culture }\end{array}$ & Not mentioned & Abortion \\
\hline
\end{tabular}

(Continued) 
Table 2 (Continued).

\begin{tabular}{|c|c|c|c|c|c|c|c|c|}
\hline $\begin{array}{l}\text { Patient } \\
\text { No. }\end{array}$ & Reference & $\begin{array}{l}\text { Age } \\
\text { (Years) }\end{array}$ & Country & Trimester & $\begin{array}{l}\text { Clinical } \\
\text { Manifestations }\end{array}$ & $\begin{array}{l}\text { Diagnostic } \\
\text { Methods }\end{array}$ & $\begin{array}{l}\text { Duration of } \\
\text { Delayed } \\
\text { Diagnosis }\end{array}$ & $\begin{array}{l}\text { Obstetric } \\
\text { Outcomes }\end{array}$ \\
\hline 31 & Sabzevari $(2018)^{46}$ & 32 & Iran & $\begin{array}{l}\text { Not } \\
\text { mentioned }\end{array}$ & $\begin{array}{l}\text { Fever, severe } \\
\text { arthralgia, chilling, } \\
\text { sweating }\end{array}$ & $\begin{array}{l}\text { Coomb's test } \\
\text { SAT } \\
\text { BAB } \\
2 M E\end{array}$ & Not mentioned & $\begin{array}{l}\text { Term } \\
\text { delivery } \\
\text { (caesarean) }\end{array}$ \\
\hline 32 & Zhao (2019) ${ }^{47}$ & 26 & China & Third & $\begin{array}{l}\text { Hepatosplenomegaly, } \\
\text { weakness in lower } \\
\text { extremities and hip } \\
\text { joint pain, fever, } \\
\text { shortness of breath }\end{array}$ & $\begin{array}{l}\text { Blood culture } \\
\text { PCR }\end{array}$ & Not mentioned & $\begin{array}{l}\text { Preterm } \\
\text { delivery }\end{array}$ \\
\hline 33 & Tian $(2019)^{48}$ & 26 & China & Second & $\begin{array}{l}\text { Intermittent fever, } \\
\text { right thigh and hip } \\
\text { pain }\end{array}$ & SAT & 5 months & $\begin{array}{l}\text { Preterm } \\
\text { delivery }\end{array}$ \\
\hline
\end{tabular}

Abbreviations: 2ME, 2-mercaptoethanol; BAB, blocking antibody titer; CFT, complement fixation test; Coomb's test, anti-human immunoglobulin test; ELISA, enzymelinked immunosorbent assay; IUFD, intrauterine fetal death; PCR, polymerase chain reaction; RBPT, rose bengal plate agglutination test; SAT, serum agglutination test.

high risk of being encountered with brucellosis in these areas. Furthermore, the incidence of pregnant women with brucellosis was $1.5 \%$ in the study by Roushan et al, ${ }^{20}$ while another study showed that the incidence of pregnant women with brucellosis was $20.6 \%$ in Iran, ${ }^{14}$ and much higher than the extracted highest incidence (5.8\%) of brucellosis occurred in pregnant women in this review. This may be explained by that incidence of human brucellosis was high during these years and there were significant differences in the geographic distribution of brucellosis in Iran. ${ }^{14,21}$ Serum agglutination test (SAT) was the most common laboratory test in the diagnosis of brucellosis in the included studies, indicating its better sensitivity or wider application compared with the culture or PCR. ${ }^{22}$

Regarding the clinical manifestations, the pregnant women with brucellosis presented as non-specific flu-like symptoms, and this may cause a huge possibility of misdiagnosis or delayed diagnosis. A total of 36 clinical manifestations were extracted from the included articles, which could provide the clinicians and the public with a clearer spectrum of the clinical manifestation of this disease. The most common clinical manifestations of pregnant women with brucellosis were fever, joint pain/swelling/arthralgia, and sweats, this is mostly consistent with some systematic reviews recording the clinical manifestations of general human with brucellosis. ${ }^{16,18}$ What is worth mentioning is that the percentage $(36.3 \%)$ of patients who had back pain among the pregnant women with brucellosis was much higher than that $(28.0 \%)$ in the general brucellosis patients, ${ }^{13}$ and as pregnancy could be accompanied with the symptoms such as sweating and back pain, the clinical manifestations of brucellosis might be misrelated to the common symptoms during pregnancy and resulted in the delay of brucellosis diagnosis. The longest speculated duration of delayed diagnosis was 5 months in the present review, and considering the fact that only a few case reports mentioned the detailed duration of delayed diagnosis, it is hard to figure out the effect of misdiagnosis or delayed diagnosis on the severity of the disease, and further exploration is needed.

The most frequently involved body system was the osteoarticular system, as joint pain was most reported, the frequency was much higher than that in some other studies about the general brucellosis patients in these areas. ${ }^{16}$ Compared with another review about clinical manifestations of brucellosis in the general population, ${ }^{16}$ the proportions of signs occurred in the gastrointestinal tract, respiratory, genitourinary, neurologic and cutaneous system in this review were much lower. With the help of the summarized wide spectrum of clinical manifestations of human brucellosis during pregnancy in the present review, brucellosis should be considered as a differential diagnosis for sweats and fevers of unknown origin.

In addition, it is still controversial about whether brucellosis can cause more serious obstetric outcomes in the pregnant women than in the general people. It was found that brucellosis might be associated with a high rate of 


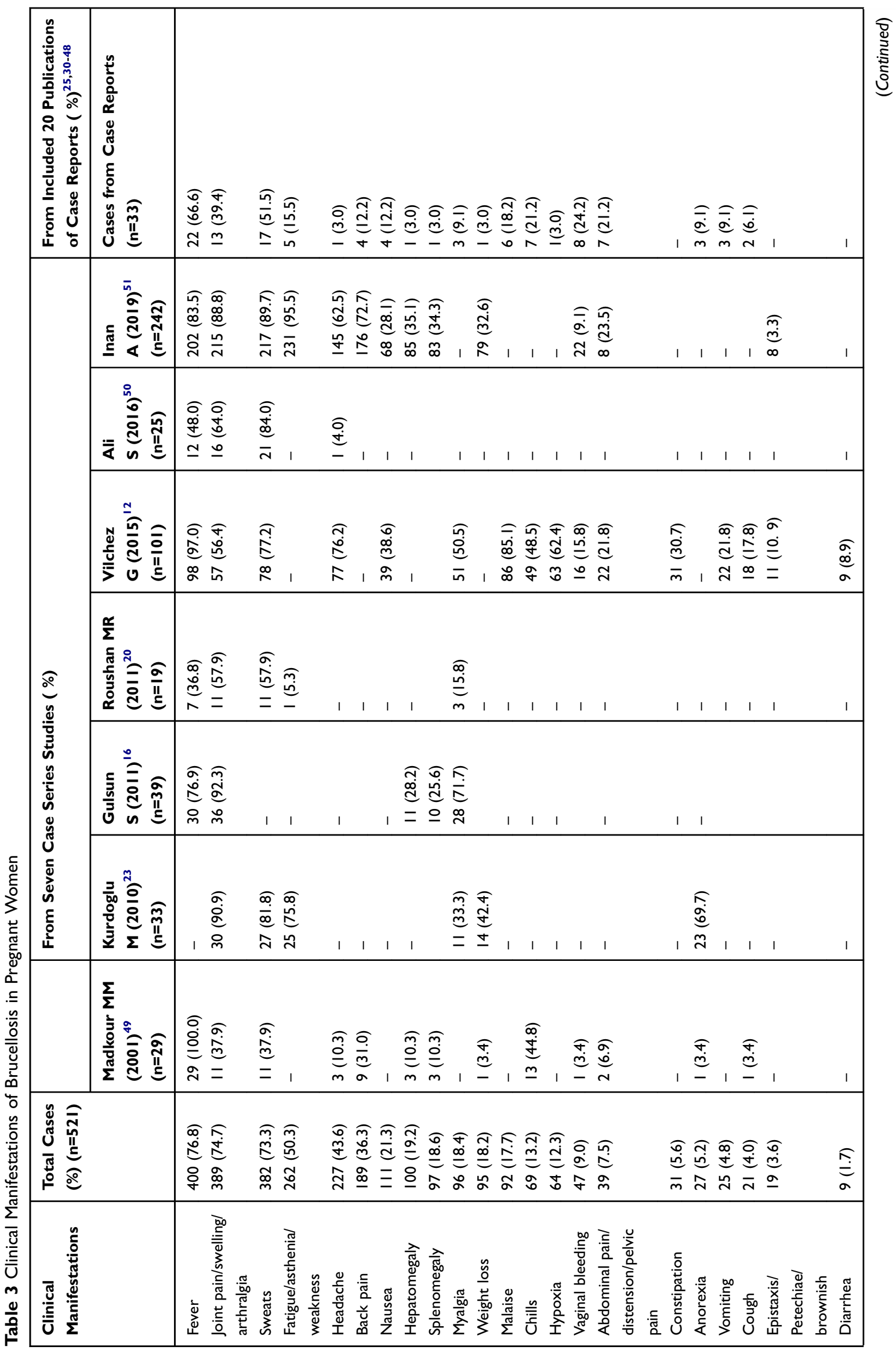




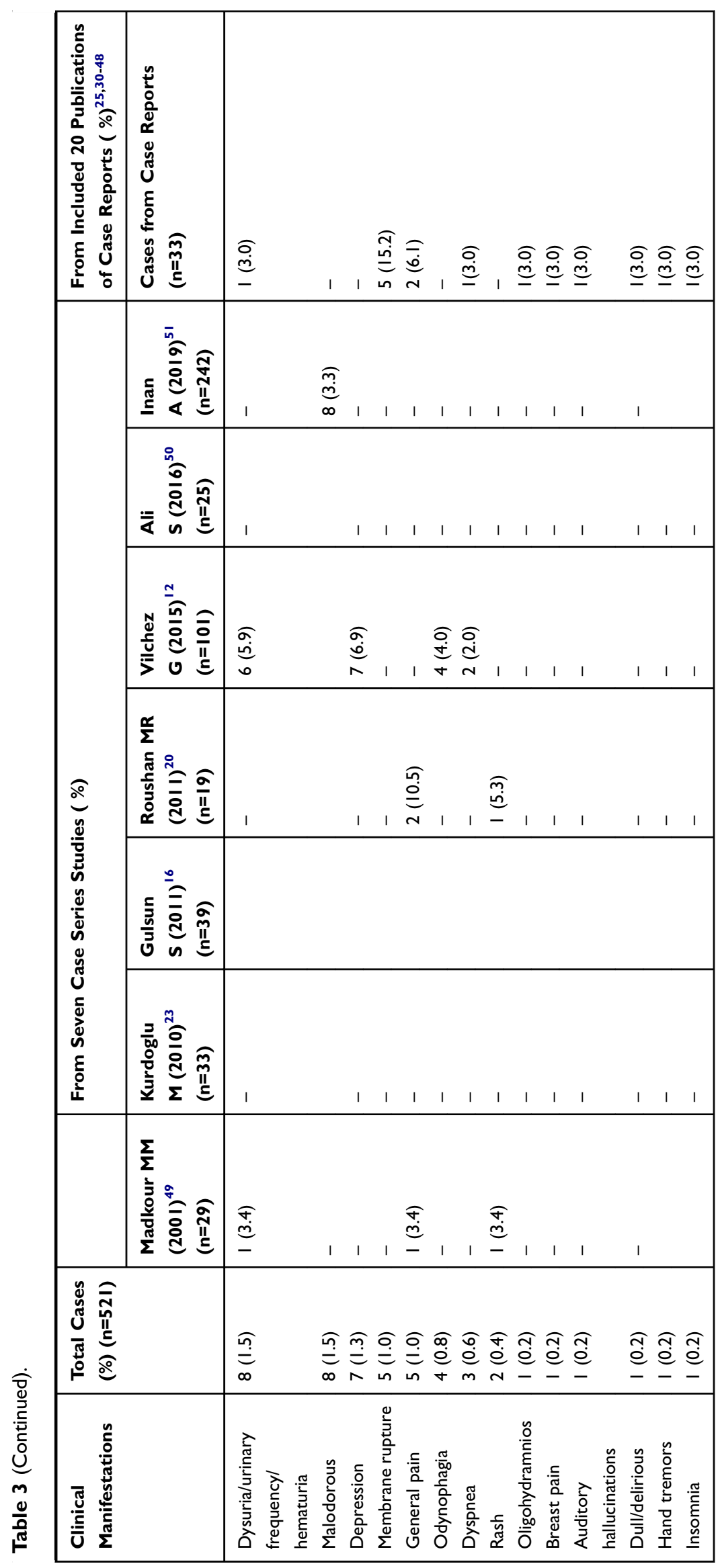


abortion or preterm delivery in some articles. ${ }^{23,24}$ Of the seven included case series, the study by Roushan et al contributed the smallest number of patients while reported the highest rate of spontaneous abortion $(52.6 \%){ }^{20}$ The included case series study by Kurdoglu et al indicated that $57.1 \%$ of the patients who presented vaginal bleeding had an abortion, which was similar as the result from the included individual case reports in the present systematic scoping review; also, it was found that vaginal bleeding was correlated highly with the pregnancy outcome with statistical significance. ${ }^{23}$ And Elshamy et al reported that pregnant women with brucellosis were more likely to have a spontaneous abortion than those pregnant women without brucellosis ${ }^{10}$; however, others did not find any correlation between antibody titer and pregnancy outcomes. ${ }^{23}$ As for the treatment, it was reported that among the 13 pregnant brucellosis cases treated with cotrimoxazole plus rifampin, $30.8 \%$ had spontaneous abortion. ${ }^{25}$ However, another study reported that antepartum antimicrobial therapy with cotrimoxazole or cotrimoxazole/rifampin was protective against spontaneous abortion, $10.0 \%$ of the 40 treated patients had spontaneous abortion or fetal death. ${ }^{9}$

Most of the included pregnant brucellosis patients were young or middle-aged, and it was obvious that the consumption of cheese or unpasteurized dairy products and stockbreeding might be the two significant risk factors of being infected with brucellosis for pregnant women, and most patients came from rural or low socioeconomic areas confessed that there had been one or more person already diagnosed as brucellosis in their family or neighbors. ${ }^{26}$ This might be attributed to the fact that family members had similar living and eating habits. It could also be speculated that the distribution of human brucellosis could be affected by the condition of poverty, educational level and occupation distribution of the population, which could be supported by the conclusion that the awareness and knowledge of brucellosis was insufficient in Asia and Africa. ${ }^{2}$

A better understanding of the potential drivers, socioeconomic predictors of human brucellosis and the disease characteristics of some specific populations is crucial to the adjustment of brucellosis control strategies and the allocation of health resources. ${ }^{27,28}$ From the above, a practical and cost-effective screening test might be helpful to identify brucellosis among family members of patients with brucellosis. And in order to prevent brucellosis for pregnant women, it was recommended that RBPT could be used as a screening test, also, the universal health education and successful animal brucellosis control or eradication programs would be the effective ways to prevent human brucellosis in endemic areas. ${ }^{29}$

The present systematic scoping review and analysis of case reports and case series had certain limitations. First, only a few case series focused on the clinical manifestations about pregnant women with brucellosis, especially focused on the differences of clinical manifestations between the pregnant women and other population. More detailed explorations into the differences between pregnant women with brucellosis and the general brucellosis patients could be conducted. Second, the spectrum of clinical manifestations of brucellosis in pregnant women remains incomplete, many complications in various body systems were not mentioned in the included articles. Thus, we suggest that the researchers can divide the clinical manifestations of brucellosis into general symptoms/ signs and localized body system complications when they conduct the descriptive study of brucellosis case report or case series in the future; this might contribute to the clearer clinical manifestation spectrum of brucellosis.

\section{Conclusion}

This systematic scoping review summarized the wide spectrum of clinical manifestations of human brucellosis in pregnancy. These various clinical manifestations would allow a better and more complete understanding of this disease for health-care providers, provide evidence for timely diagnosis, adequate therapy and better prevention, and might reduce doctors' misdiagnoses.

\section{Acknowledgments}

This work was supported by the National Natural Science Foundation of China (Grants No: 71974199 and 71573275) and Science Foundation of Liaoning Provincial Department of Education (LFWK201719 and JC2019036). The findings and conclusions in this review are those of the authors and the funders had no role in study design, data collection and analysis, decision to publish, or preparation of the manuscript.

\section{Disclosure}

The authors declare no conflicts of interest in this work.

\section{References}

1. World Health Organization. The Control of Neglected Zoonotic Diseases: A Route to Poverty Alleviation. Geneva World Health Organization; 2006. 
2. Zhang N, Zhou H, Huang DS, Guan P. Brucellosis awareness and knowledge in communities worldwide: a systematic review and meta-analysis of 79 observational studies. PLoS Negl Trop Dis. 2019;13(5):e0007366. doi:10.1371/journal.pntd.0007366

3. De Massis F, Di Girolamo A, Petrini A, Pizzigallo E, Giovannini A. Correlation between animal and human brucellosis in Italy during the period 1997-2002. Clin Microbiol Infect. 2005;11(8):632-636. doi:10.1111/j.1469-0691.2005.01204.x

4. Mangalgi SS, Sajjan AG, Mohite ST, Gajul S. Brucellosis in occupationally exposed groups. J Clin Diagn Res. 2016;10(4):DC24DC27. doi:10.7860/JCDR/2016/15276.7673

5. Tuon FF, Gondolfo RB, Cerchiari N. Human-to-human transmission of Brucella - a systematic review. Trop Med Int Health. 2017;22 (5):539-546. doi:10.1111/tmi. 12856

6. Yagupsky P. Neonatal brucellosis: rare and preventable. Ann Trop Paediatr. 2010;30(3):177-179. doi:10.1179/146532810X127863889 78445

7. Godfroid J, Cloeckaert A, Liautard JP, et al. From the discovery of the Malta fever's agent to the discovery of a marine mammal reservoir, brucellosis has continuously been a re-emerging zoonosis. Vet Res. 2005;36(3):313-326. doi:10.1051/vetres:2005003

8. Eyre JWH. The milroy lectures on melitensis septicæmia (malta or mediterranean fever). Lancet. 1908;171(4424):1826-1832. doi:10.10 16/S0140-6736(01)64377-0

9. Khan MY, Mah MW, Memish ZA. Brucellosis in pregnant women. Clin Infect Dis. 2001;32(8):1172-1177. doi:10.1086/319758

10. Elshamy M, Ahmed AI. The effects of maternal brucellosis on pregnancy outcome. J Infect Dev Ctries. 2008;2(3):230-234. doi:10. 3855/jidc. 268

11. Rujeni N, Mbanzamihigo L. Prevalence of brucellosis among women presenting with abortion/stillbirth in Huye, Rwanda. J Trop Med. 2014;2014:740479. doi:10.1155/2014/740479

12. Vilchez G, Espinoza M, D’Onadio G, Saona P, Gotuzzo E. Brucellosis in pregnancy: clinical aspects and obstetric outcomes. Int J Infect Dis. 2015;38:95-100. doi:10.1016/j.ijid.2015.06.027

13. Buzgan T, Karahocagil MK, Irmak H, et al. Clinical manifestations and complications in 1028 cases of brucellosis: a retrospective evaluation and review of the literature. Int J Infect Dis. 2010;14(6):e469e478. doi:10.1016/j.ijid.2009.06.031

14. Ahmadi A, Mohsenpour B, Doroudian P, et al. A seroprevalence and relationship survey of brucellosis between pregnant women and women with spontaneous abortion in Iran. Med J Islam Repub Iran. 2017;31(1):42. doi:10.14196/mjiri.31.42

15. Young EJ. Human Brucellosis. Rev Infect Dis. 1983;5(5):821-842. doi:10.1093/clinids/5.5.821

16. Gulsun S, Aslan S, Satici Omer S, Gul T. Brucellosis in pregnancy. Trop Doct. 2011;41(2):82-84. doi:10.1258/td.2011.100386

17. Moher D, Liberati A, Tetzlaff J, Altman DG; The PRISMA Group. Preferred reporting items for systematic reviews and meta-analyses: the PRISMA statement. PLoS Med. 2009;6(7):e1000097. doi:10.13 71/journal.pmed1000097

18. Dean AS, Crump L, Greter H, Hattendorf J, Schelling E, Zinsstag J. Clinical manifestations of human brucellosis: a systematic review and meta-analysis. PLoS Negl Trop Dis. 2012;6(12):e1929. doi:10.1371/ journal.pntd.0001929

19. Dean AS, Crump L, Greter H, Schelling E, Zinsstag J, Carabin H. Global burden of human brucellosis: a systematic review of disease frequency. PLoS Negl Trop Dis. 2012;6(10):e1865. doi:10.1371/journal.pntd.0001865

20. Roushan MR, Baiani M, Asnafi N, Saedi F. Outcomes of 19 pregnant women with brucellosis in Babol, northern Iran. Trans R Soc Trop Med Hyg. 2011;105(9):540-542. doi:10.1016/j.trstmh. 2011;06.003

21. Pakzad R, Pakzad I, Janati A, et al. Spatiotemporal analysis of brucellosis incidence in Iran from 2011-2014 using GIS. Int J Infect Dis. 2017;67:129-136. doi:10.1016/j.ijid.2017.10.017
22. Marei A, Boghdadi G, Abdel-Hamed N, et al. Laboratory diagnosis of human brucellosis in Egypt and persistence of the pathogen following treatment. J Infect Dev Ctries. 2011;5(11):786-791. doi:10.38 55/jidc. 1538

23. Kurdoglu M, Adali E, Kurdoglu Z, et al. Brucellosis in pregnancy: a 6-year clinical analysis. Arch Gynecol Obstet. 2010;281(2):201-206. doi:10.1007/s00404-009-1106-0

24. Seoud M, Saade G, Awar G, Uwaydah M. Brucellosis in pregnancy. J Reprod Med. 1991;36(6):441-445. doi:10.1258/td.2011.100386

25. Schreyer P, Caspi E, Leiba Y, Eshchar Y, Sompolinsky D. Brucella septicemia in pregnancy. Eur J Obstet Gynecol Reprod Biol. 1980;10 (2):99-107. doi:10.1016/0028-2243(80)90087-8

26. Kledmanee K, Liabsuetrakul T, Sretriritchai S. Risk of adverse pregnancy outcomes and seroprevalence for brucellosis in pregnant women exposed to goats or raw goat products in Southern Thailand: a prospective cohort study. BMC Pregnancy Childbirth. 2019;19(1):118. doi:10.1186/s12884-019-2267-x

27. Peng C, Li YJ, Huang DS, Guan P. Spatial-temporal distribution of human brucellosis in mainland China from 2004 to 2017 and an analysis of social and environmental factors. Environ Health Prev Med. 2020;25(1):1. doi:10.1186/s12199-019-0839-Z

28. Peng C, Zhou H, Guan P, Wu W, Huang DS. An estimate of the incidence and quantitative risk assessment of human brucellosis in mainland China. Transbound Emerg Dis. 2020. doi:10.1111/tbed.13 518

29. Zhang N, Huang D, Wu W, et al. Animal brucellosis control or eradication programs worldwide: a systematic review of experiences and lessons learned. Prev Vet Med. 2018;160:105-115. doi:10.1016/j. prevetmed.2018.10.002

30. Sarram M, Feiz J, Foruzandeh M, Gazanfarpour P. Intrauterine fetal infection with Brucella melitensis as a possible cause of second-trimester abortion. Am J Obstet Gynecol. 1974;119(5):65 7-660. doi:10.1016/0002-9378(74)90128-8

31. Naveau S, Poitrine A, Delfraissy JF, Brivet F, Dormont J. Brucellosis hepatic abscesses and pregnancy. Gastroenterology. 1983;84(6):1643. doi:10.1016/0016-5085(83)90420-1

32. Gloeb DJ, Lupi C, O’Sullivan MJ. Neurobrucellosis complicating pregnancy: a case report. Infect Dis Obstet Gynecol. 1994;1 (6):285-289. doi:10.1155/S1064744994000268

33. Dan CJ, Ge HC. Reports and analysis of two cases of pregnancy with brucellosis. Chin J Obstet Gynecol. 2001;36(5):304. (Article in Chinese).

34. Jensenius M, von der Lippe B, Hermansen NO, et al. Brucellar mastitis: presentation of a case and review of the literature. Int J Infect Dis. 2008;12(1):98-100. doi:10.1016/j.ijid.2007.03. 007

35. Cebesoy FB, Balat O, Mete A. An extraordinary cause of vertebral fracture in pregnant woman: brucellosis. Arch Gynecol Obstet. 2009;280(2):301-303. doi:10.1007/s00404-008-0887-x

36. Karcaaltincaba D, Sencan I, Kandemir O, Guvendag-Guven ES, Yalvac S. Does brucellosis in human pregnancy increase abortion risk? Presentation of two cases and review of literature. J Obstet Gynaecol Res. 2010;36(2):418-423. doi:10.1111/j.1447-0756.2009. 01156.x

37. Peker N, Turan V, Ergenoglu M, Yeniel O. Brucellosis in adolescent pregnancy-case report and review of literature. Ginekol Pol. 2011;82 (3):226-229. doi:10.1111/j.1447-0756.2009.01156.x

38. Ceylan A, Köstü M, Tuncer O, Peker E, Kırımi E. Neonatal brucellosis and breast milk. Indian J Pediatr. 2012;79(3):389-391. doi:10. 1007/s12098-011-0581-z

39. Bi XQ, Zhao C, Wei LH. One case reports of brucellosis during pregnancy. Chin J Clin Obstet Gynecol. 2013;14(4):367-368. (Article in Chinese).

40. Aydin B, Beken S, Akansel R, et al. Prematurity due to maternal brucella infection and review of the literature. Turk $J$ Pediatr. 2013;55(4):433-437. 
41. Li X, Chang LY. One case of abortion caused by Brucella maltatis. Xinjiang Med J. 2015;45(12):1812-1813. (Article in Chinese).

42. Fatnassi R, Marwen N, Benltaifa A, Barhoumi T. Specificity of brucellosis in pregnancy: presentation of two cases and review of literature. J Infect Dis Ther. 2016;4:308. doi:10.4172/2332-0877. 1000308

43. Agah J, Jafarzadeh Esfehani R, Kamalimanesh B. Different manifestation of brucellosis in pregnancy: case reports. $J$ Microbiol Infect Dis. 2016;6:80-83. doi:10.5799/ahinjs.02.2016.02.0221

44. Zhou JW, Guo ZY, Zhu X, Yan ST, Li TC. Two case reports of pregnancies with Brucellosis. Henan J Prev Med. 2017;28(2):15 9-161. (Article in Chinese).

45. Yang HX, Feng JJ, Zhang QX, et al. A case report of spontaneous abortion caused by Brucella melitensis biovar 3. Infect Dis Poverty. 2018;7(1):31. doi:10.1186/s40249-018-0411-x

46. Sabzevari S, Badirzadeh A, Kalateh A, Seyyedin M. Development of severe fever due to brucellosis after Cesarean: case report from Iran $J$ Case Rep. 2018;8(1):1-3. doi:10.17659/01.2018.0001
47. Zhao M, Huang F, Zhang A, et al. Congenital brucellosis in a Chinese neonate: a case report. $J$ Int Med Res. 2019;47(5):2296-2301. doi:10. $1177 / 0300060519838921$

48. Tian G, Zhan Z, Zhang A, et al. A case report on mother-to-child transmission of Brucella in human, China. BMC Infect Dis. 2019;19 (1):666. doi:10.1186/s12879-019-4302-y

49. Madkour MM. Pregnancy and Brucellosis. Madkour's Brucellosis. Berlin Heidelberg: Springer; 2001. doi:10.1007/978-3-642-59533217

50. Ali S, Akhter S, Neubauer H, et al. Brucellosis in pregnant women from Pakistan: an observational study. BMC Infect Dis. 2016;16:468. doi:10.1186/s12879-016-1799-1

51. Inan A, Erdem H, Elaldi N, et al. Brucellosis in pregnancy: results of multicenter ID-IRI study. Eur J Clin Microbiol Infect Dis. 2019;38 (7):1261-1268. doi:10.1007/s10096-019-03540-z
Infection and Drug Resistance

\section{Publish your work in this journal}

Infection and Drug Resistance is an international, peer-reviewed openaccess journal that focuses on the optimal treatment of infection (bacterial, fungal and viral) and the development and institution of preventive strategies to minimize the development and spread of resistance. The journal is specifically concerned with the epidemiology of
Dovepress

antibiotic resistance and the mechanisms of resistance development and diffusion in both hospitals and the community. The manuscript management system is completely online and includes a very quick and fair peerreview system, which is all easy to use. Visit http://www.dovepress.com/ testimonials.php to read real quotes from published authors. 